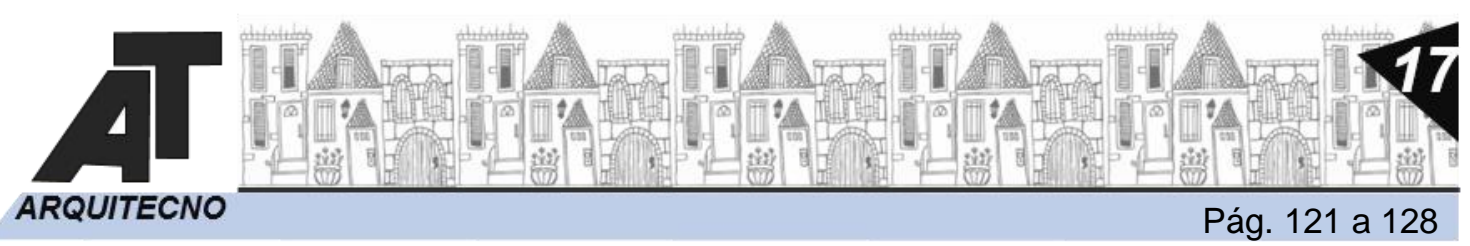

\title{
Segunda Vida. \\ Construcción a partir de elementos recuperados
}

\author{
Second Life. \\ Construction from Recovered Items
}

Edgardo Lufiego y Rocío Lufiego

Taller de Producción de Obras/ Oficina de Materiales / LFG la Plata / SisedLab / Facultad de Arquitectura-Universidad Nacional de La Plata

E-mail de contacto_lfglaplata@gmail.com, lufiego@hotmail.com

\section{RESUMEN}

Segunda Vida

La investigación en materiales y tecnología se orienta a la recuperación de materiales de desechos o de descarte para ser utizados en la construcción, en particular para sectores de bajos recursos, que no podrían acceder a viviendas por otros medios o al completamiento de las mismas.

Reciclando Tarimas o Pallets de madera, recuperados como residuos de obras de construcción, deconstruyéndolos o desarmando, obteniendo componentes. Madera. Panelizando luego con esos materiales tanto paneles verticales como paneles de techo, autoportantes.

Paralelamente sobre todo en medio de la Pandemia del COVID 19 hemos estado viendo nuevos usos para esos mismos materiales recuperados, destinados a una población con distintas necesidades, ya no con la urgencia de vivienda y techo sinó como complementos, mobiliario y/o construcciones menores. Que no obstante pueden permitir una salida laboral viable.

\section{ABSTRACT}

Second Life

Research in materials and technology is aimed at the recovery of waste or discarded materials to be used in construction, particularly for low-income sectors, who could not access housing by other means or to complete them.

Recycling pallets or wooden pallets, recovered as construction site waste, deconstructing or disassembling them, obtaining components. Wood. Then panelizing with these materials both vertical panels and ceiling panels, self-supporting.

In parallel, especially in the midst of the COVID 19 Pandemic, we have been seeing new uses for those same recovered materials, destined for a population with different needs, no longer with the urgency of housing and roof but as accessories, furniture and / or minor constructions. That nonetheless can allow a viable job opportunity.

PALABRAS CLAVES: Reutilización, tarimas, descartadas, madera

KEY WORDS: Reuse, pallets, discarded, wood.

FECHA DE RECEPCIÓN: 31/3/2021 | FECHA DE ACEPTACIÓN: 13/5/2021

DOI: http://dx.doi.org/10.30972/arq.0174995 


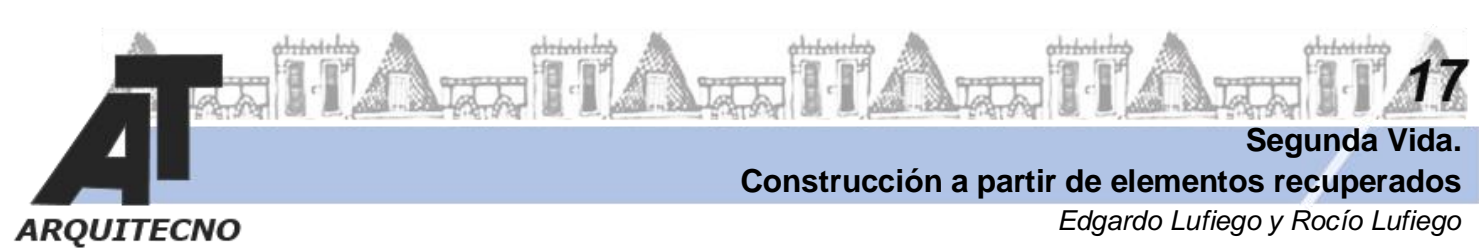

\section{INTRODUCCIÓN}

La búsqueda se orienta a los materiales recuperables.

En el marco del proyecto de investigación denominado: Contribución a la formación de criterios sustentables en la construcción y producción de espacios habitables. Facultad de Arquitectura y Urbanismo, Universidad Nacional de la Plata, Laboratorio de Investigación en Sistemas Edilicios - SISEDLAB. Proyecto I+D 11-U177.

Vivimos en un momento en que se ha convertido en algo indispensable ser parte, desde la arquitectura, de un un modelo de crecimiento sostenible o sustentable Se pretende integrar al diseño arquitectónico elementos basados en el respeto al medioambiente y en la implementación de sistemas de eficiencia energética.

Nuestro punto de vista es tratar cuestiones como el estudio de materiales, la elaboración de herramientas tanto digitales como analógicas, criterios específicos de diseño, que den soporte en diseño sustentable, a las actividades pedagógicas relacionadas con la formación del estudiante (y del graduado).

Temáticas abordadas: arquitectura sustentable, tecnologia y materiales.

La búsqueda desde la oficina de materiales esta orientada a materiales y tecnologías como componentes y o sistemas para la construcción de muy bajo costo (tendiendo a cero).

Apelando a la reutilización, reciclado y recuperación de materiales de descarte.

Probando y experimentando distintas opciones y variables.

Desde la producción de bloques a partir de envases PET recuperados, o placas a partir de polímeros de tapas de gaseosas o multiples capas de cartón, pegadas y prensadas.

Sin embargo, estos desarrollos terminaban produciendo componentes menores, una placa, un ladrillo, etc, que si bien reciclaban y se producían a un costo bajo no daban una solución integral a la necesidad de vivienda detectada.

De las distintas locaciones relevadas tanto en la zona La Plata y gran La Plata como en distintos barrios y asentamientos en el conurbano y municipios vecinos, V.G. Alejandro Korn, San Vicente etc. pudimos:

1) Asimilar las construcciones locales a sistemas constructivos con estructura de madera tipo entramados, ballon frame, y por otro lado vimos como una necesidad dar una pronta respuesta a necesidades de cerramiento, fundamentalmente horizontal y vertical Techo y pared.

2) Detectar como elementos de desecho, o de descarte, una cantidad importante de Pallets o tarimas de madera, que se encontraban directamente en las veredas, o en los contenedores donde se vierten los escombros y sobrantes de las obras en construcción.

Uniendo estas 2 variables es que redireccionamos nuestra actividad de Investigación, apuntalándola luego en las Actividades de Grado y en las de Extensión.

Centrándonos en la recuperación de madera de pallets para elaborar paneles para la envolvente de una construcción como muros y cubiertas.

Entendiendo la necesidad de la interacción entre las tres actividades que son pilares de nuestra Universidad, Docencia, Investigación y Extensión. 
Ante la necesidad detectada en los estudios de campo realizados y desarrollados con la participación de los alumnos en las actividades de grado y en las actividades de extensión llevadas a cabo.

Se ve la necesidad de poder prover de sistemas o componentes para el mejoramiento, 0 ampliación o construcción de viviendas o módulos habitables.

\section{METODOLOGÍA}

\section{Características ó tipologías de los elementos residuales.}

En general la madera utilizada es Saligna sin cepillar.

Si bien existen otras caracterizaciones y posibles clasificaciones nos resulta viable encuadrarlos en 2 tipos pesados y livianos.

Siendo los primeros utilizados en general en la industria de la construcción para distribución de bolsas de Cemento y/o de Cal, también se utilizan para transportar mampuestos pesados como bloques de hormigón o cerámicos o pisos de piedra etc, colocados sobre los mismos, luego los retiran de la obra, algunos se venden.

Están formados usualmente por tres tirantes o cabios de 2"x 4" $(5 \times 10 \mathrm{~cm})$

Y tablas a ambos lados de 1" x 4" $(2,5 \times 10 \mathrm{~cm})$ en general bastante juntas. Las dimensiones oscilan entre 1 y $1,20 \mathrm{~m}$.

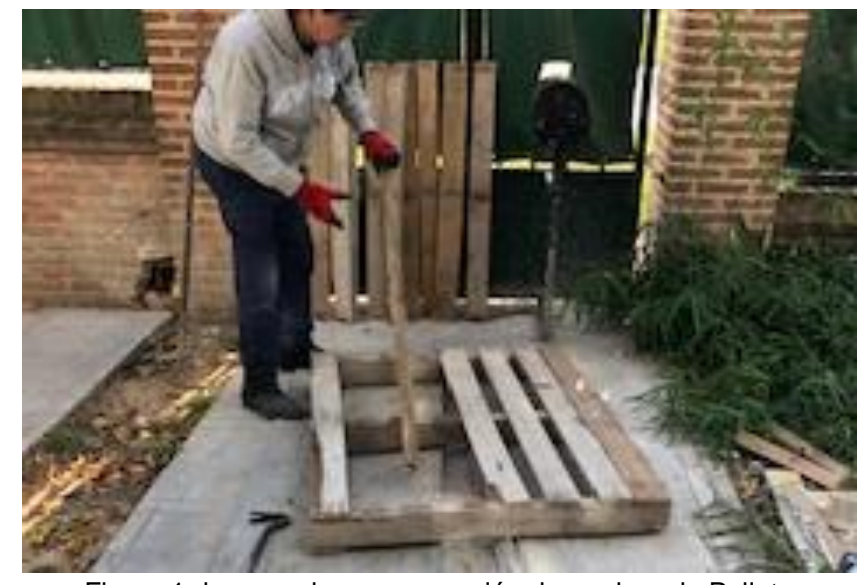

Figura 1 desarmado y recuperación de madera de Pallets

A veces se reemplazan según el fabricante los tirantes por 3 tacos de 3"x 3 " de unos $15 \mathrm{~cm}$ de largo cada uno.

Estos son los Pallets con los que se realizan trabajos de bricolaje, muebles de patio o de interior, jardines o huertas verticales etc.

Ofrecen dificultad en el desarmado. Como puede visualizarse en la Figura 1 Desarmado y recuperación de Pallet Pesado

Por el contrario, los livianos son utilizados para el embalaje y despacho a obra de ladrillos y siendo el número disponible mayor ya que debido a su bajo costo se los descarta en la obra. Siendo que no es inmediato su uso y que el acopio en obra se realiza apilando varios de estos paquetes, incluyendo el pallet.

Constituido por 3 listones de 1 " x 3 " $(2,5$ x $7 \mathrm{~cm})$ de un largo nominal de $1,00 \mathrm{~m}$ y 6 tablillas de $1 / 2$ " $\times 2 "(1 \mathrm{~cm} \times 5 \mathrm{~cm})$ de similar largo.

El amado es por clavado siendo una tarea sencilla el desarmado. 


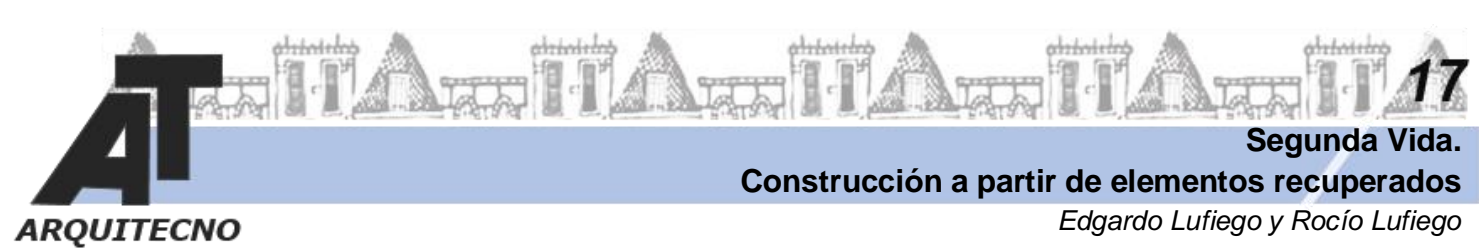

\section{Disponibilidad, Elementos ¿realmente hay tal disponibilidad como presumimos?}

Considerando las estadísticas en base a obras realizadas en la zona algunas propias y otras ajenas, pudimos establecer que tentativamente en una obra con sistema constructivo tradicional y dependiendo del diseño de la misma consume 1 pallet de ladrillos cada $4 \mathrm{~m}^{2}$ de obra.

Podríamos decir que en una obra de $200 \mathrm{~m}^{2}$ podríamos recuperar unos 50 pallets livianos. Por lo cual entendemos de amplia disponibilidad, como ilustra la figura 2 Pallets descartados.

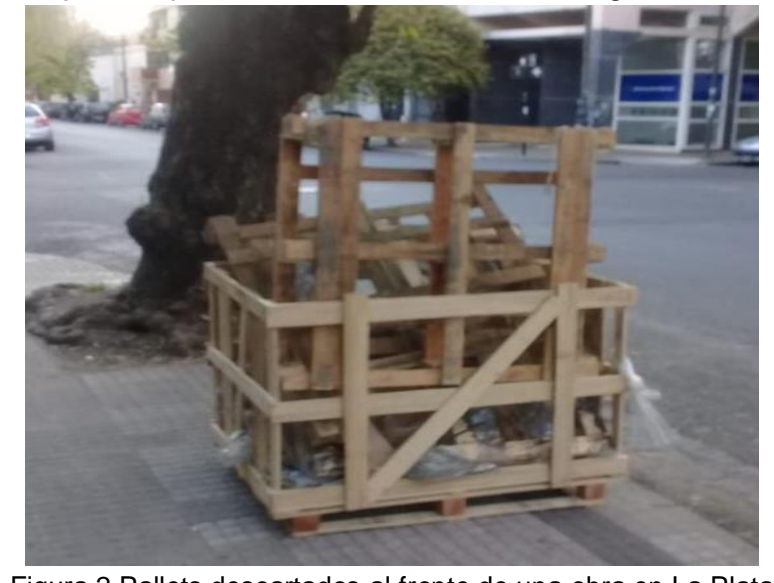

Una idea pendiente es solicitar a las autoridades municipales que soliciten a las obras al aprobarse la documentación, el guardado de los pallets para pasar a retirarlos en forma oportuna.

\section{DESARROLLO}

Que tecnología, herramientas se necesitan para realizar los trabajos.

La elección de pallets livianos para el desarrollo se fundamenta en la gratuidad de los mismos, en la cantidad disponible, y en las herramientas de mano básicas para su desarme o deconstrucción y en el armado.

Siendo herramientas básicas un martillo galponero, con uña saca clavos, y una maza como apoyo para desarmar y como "aguantador" para clavar de lado. La propuesta prevé utilizar mayormente maderas sin cortar, de todos modos es posible necesitar para preparar algunas piezas menores contar con un serrucho de mano. Ademas de elementos de medición cinta métrica regular de $3 \mathrm{~m}$.

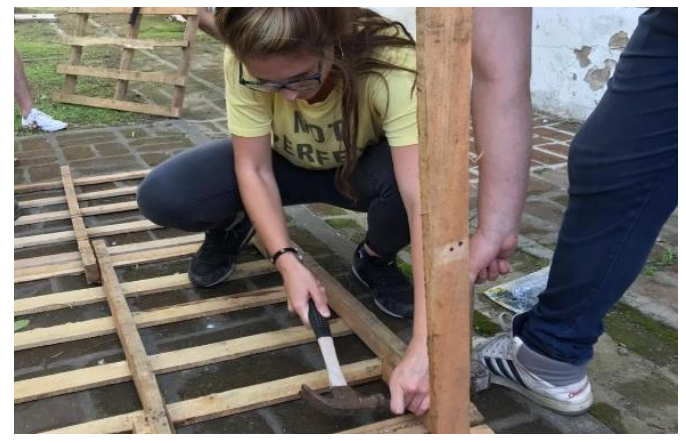

Figura 3 Panelizado realizando las uniones por clavado Las uniones se realizan por clavado con clavos de 1,1/2" Punta París, para las tablillas y de 2" Punta París, para unir llistones. (ver Figura 3 clavado por Cano de Obra no calificada) Los materiales recuperados admiten distintas terminaciones. 
Rustica como se recupera admite pintado impermeabilización por aceite de lino, aceite quemado de auto, pintura al latex.

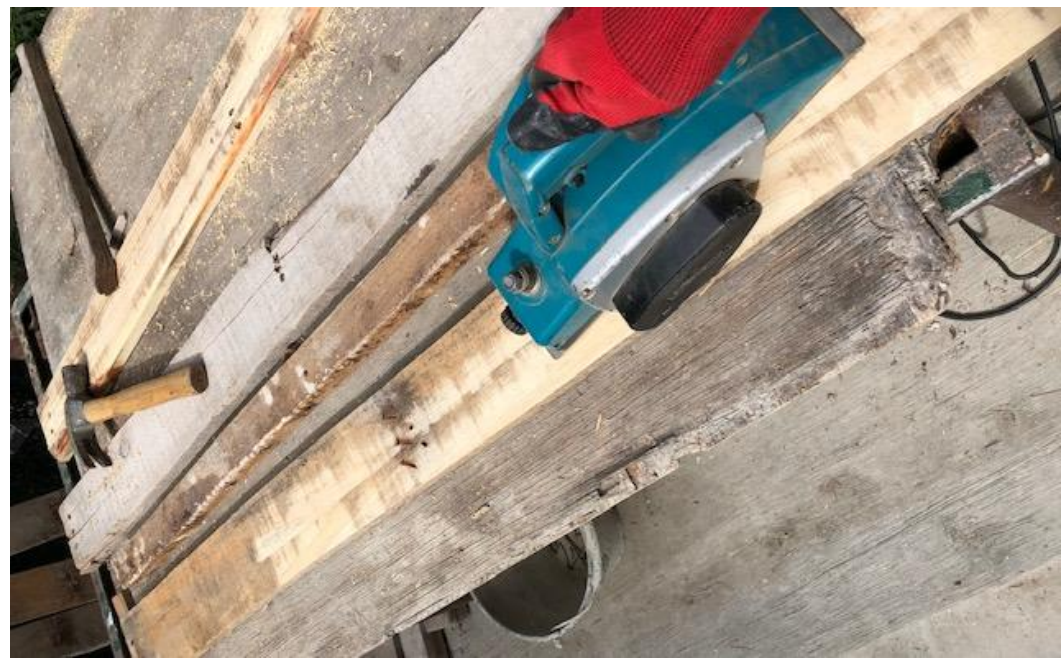

Figura 4 cepillado de madera recuperada

Admitiendo también ser cepilladas manualmente y/o lijadas en el caso de utilizarlas para construcciones complementarias o buscar otra terminación. En la Fig.4 cepillado con cepilladora eléctrica de mano

\section{Sistema constructivo asimilable a construcción en seco.}

\section{Panelización}

La propuesta se estructura en la producción de paneles utilizando los componentes de los pallets que se deconstruyen previamente, realizando la estructura de los mismos utilizando 3 listones superpuestos unidos por clavado. De un largo de 2,40 m. el panel mide 1,00 m de ancho por 2,40 m de largo constituido por 3 largueros estructurales y revestido por tablillas en una de las caras. Como observamos en la Fig. 5

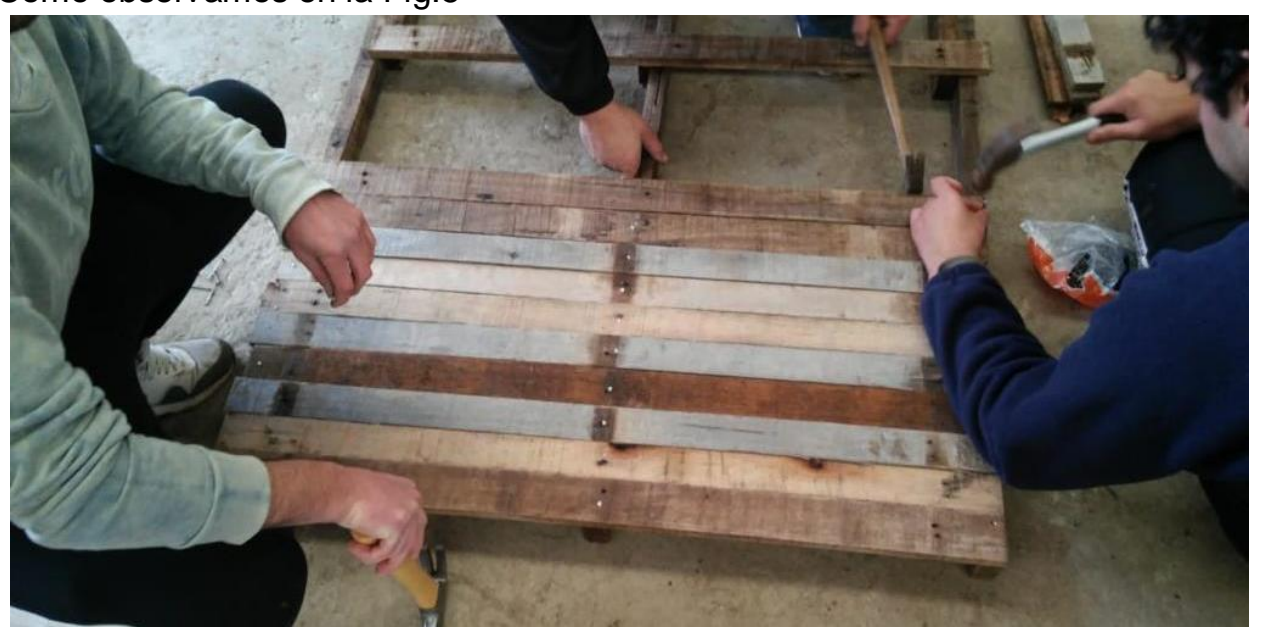

Figura 5 panelizado con tablillas

Yuxtapuestas para estructuras de techo y superpuestas para cerramientos verticales, denominado tingladillo o "siding" un sistema típico de construcción en madera.

Para la cubierta se utiliza un panel ciego de cierre constituyendose en una estructura reticulada tipo cabriada autoportante.

Condiciones alcanzadas. 


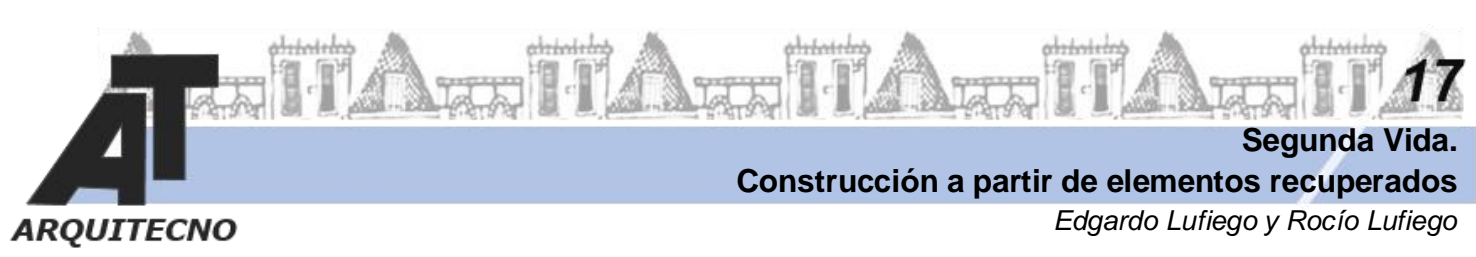

La estructura desarrollada resulta suficiente para condiciones de construcción autoportante en planta baja, y permite el armado sencillo de módulos habitables.

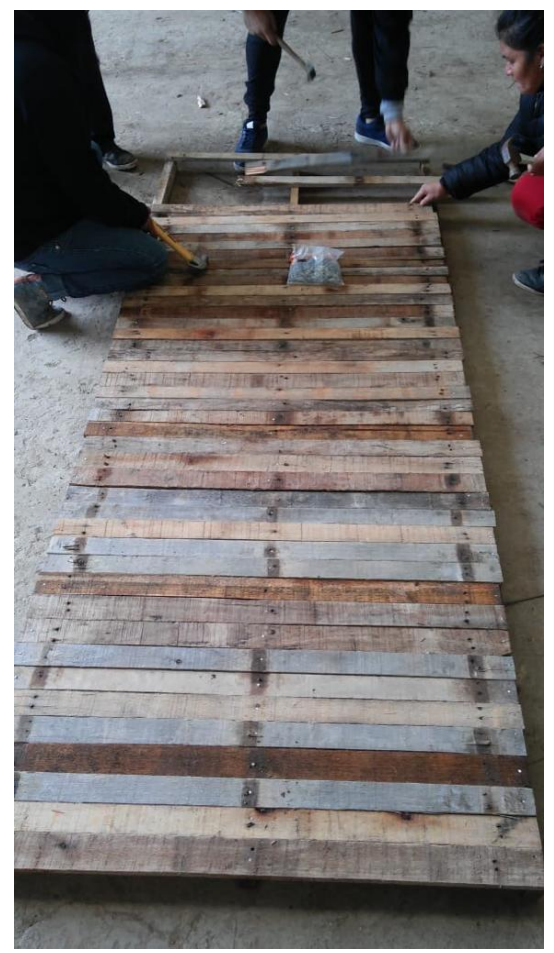

Antes de realizar la terminación interior se adicionará aislación hidrófuga y térmica por medio de la utilización de fieltro asfáltico y poliestireno expandido recuperado de embalajes.

La terminación interior podrá ser tanto de madera de tablillas, como de otros materiales según la disponibilidad del usuario.

El panel así descripto cumple con las condiciones mínimas de aislación térmica (coeficiente $\mathrm{K}$ admisible)

En el caso del panel de techo se prevé el cierre por debajo de la estructura constituyendo una suerte de cielorraso dejando una cámara de aire por encima.

La estructura así resuelta permite una variedad de cubiertas. En la fig.6 observamos un panel cubierto de Tablillas.

Figura 6 Panel $1,00 \times 2,40 \mathrm{~m}$

\section{Tiempo de ejecución.}

La realización de un módulo de techo tal como se indica en los 5 pasos del instructivo desarrollado, (Ver figura 7) pudo ser realizado con mano de obra no calificada, por 8 personas en un lapso de 2 hs 30 min., incluyendo el desarmado o deconstrucción de los pallets, la capacitación y la realización del módulo de $1 \mathrm{~m}$ x 2,40 m con 1m de altura.
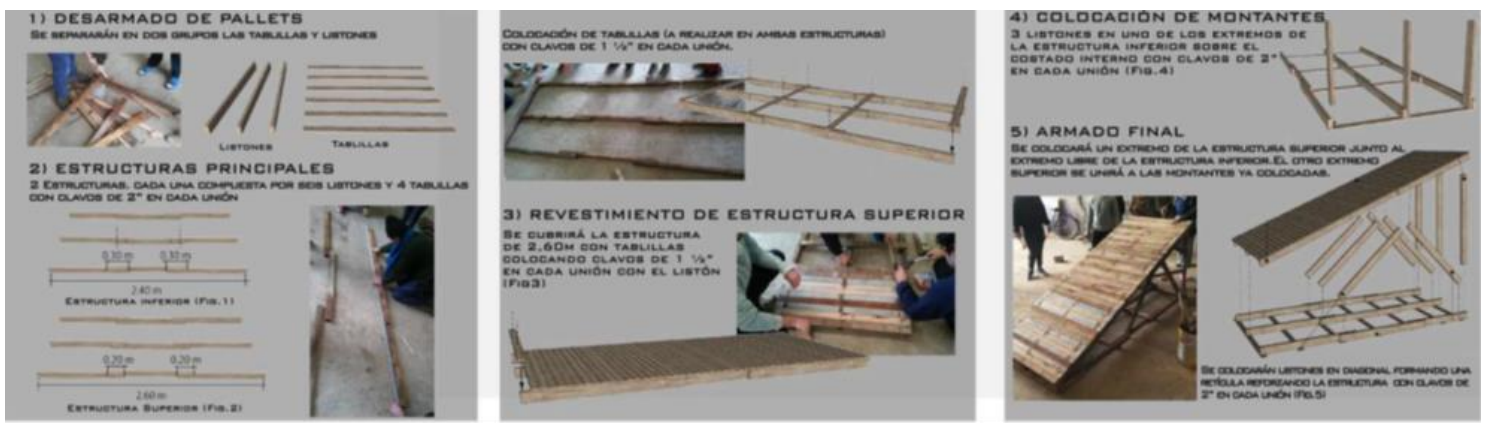

Figura 7 instructivo de armado de Techo

Por ende, resulta en una de las fortalezas la rapidez de ejecución. Resultando particularmente atractivo para la continuidad en el tiempo la pronta visibilización.

\section{DISCUSIÓN DE RESULTADOS}

Formas de difusión de los resultados y capacitación previstas. 
La idea rectora es que la investigación llevada a cabo pueda ser transferida rápidamente y a su vez retroalimente la misma. De allí la importancia de que interactúen la investigación, la docencia en el grado, como la extensión.

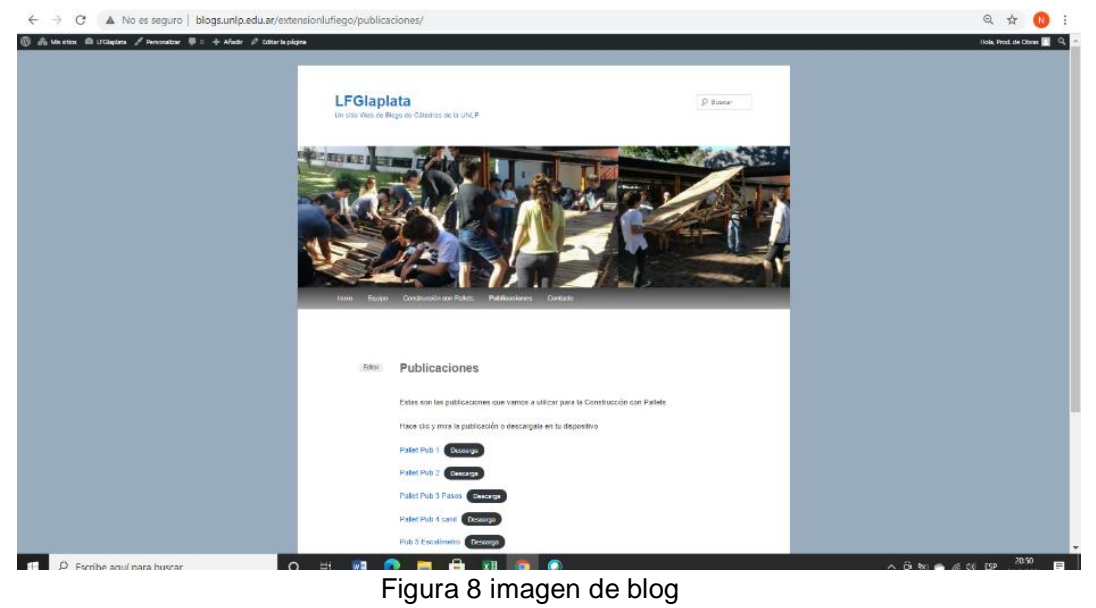

Es por ello que se están realizando una serie de publicaciones a modo de fascículos que pueden constituirse en capítulos de una publicación mayor.(Vamos actualmente por la Pub. 10)

Siendo accesibles y pudiendo descargarse o leerse el línea desde nuestro blog. LFG LaPlata (http://blogs.unlp.edu.ar/extensionlufiego/publicaciones/) (imagen del Blog en Fig. 8)

También las fotografías de los producidos pueden encontrarse en Instagram como @lfglaplata. Ver figura 9
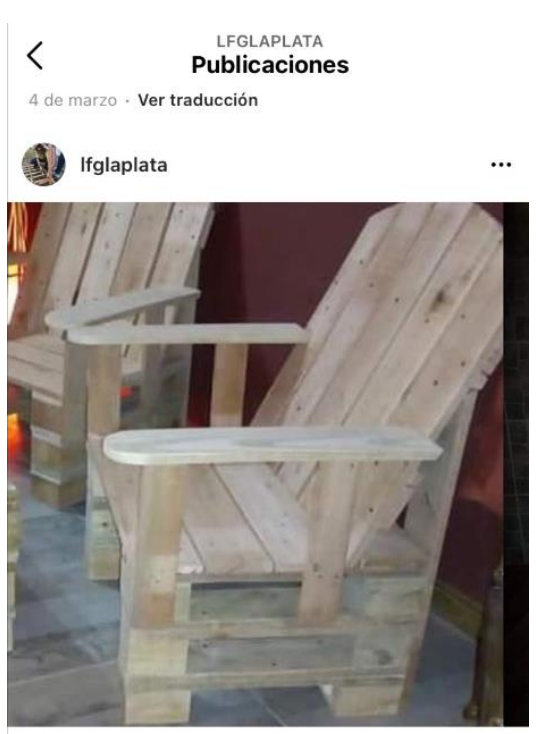

$0 \bigcirc \nabla$

3.9. Les gusta a arqurrutiamarcelo y 8 más Ifglaplata Construcción con PALLETS.

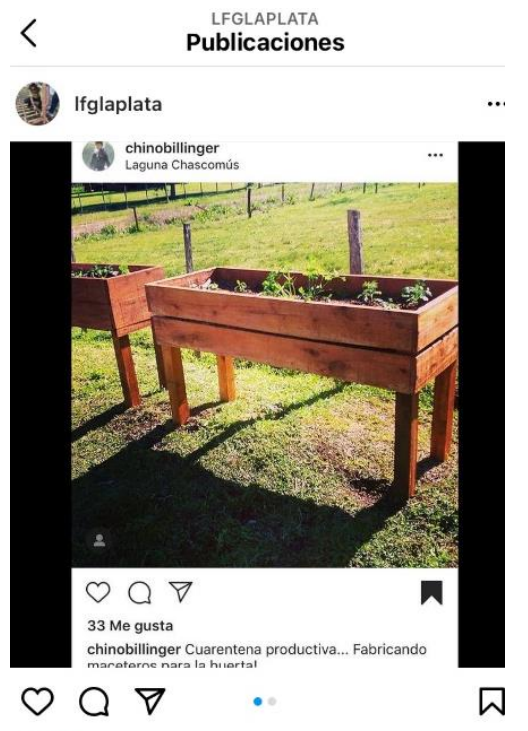

QD Les gusta a valeria.palma.c90 y 6 más Ifglaplata Construcción con PALLETS Chinobillinger de Cahscomús nos muestra sus creaciones 17 de seotiembre de 2020 . Ver traducción
ऽ
$\oplus$ ๑
(1)
๙
$\bigoplus$
仓 (19)

Figura 9 mobiliario elaborado a partir de elementos recuperados de Pallets (disponible en Instagram @lfglaplata) 


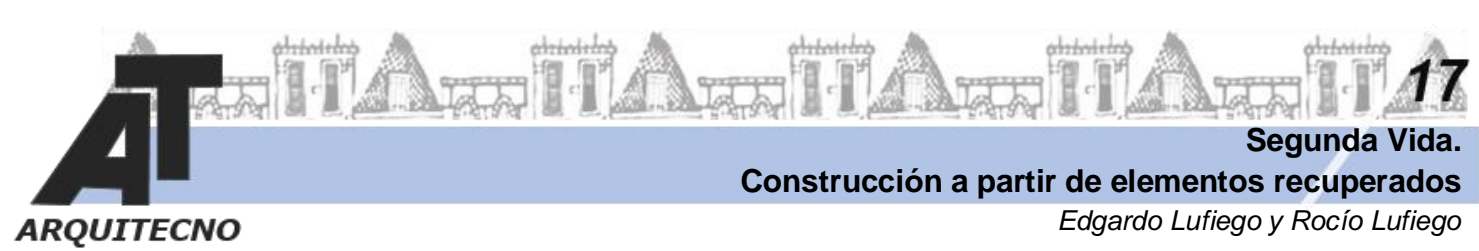

\section{CONCLUSIONES}

Queda pendiente la construcción de un módulo habitable completo, que estaba previsto realizarse durante el ciclo lectivo 2020, que no pudo realizarse por la pandemia del COVID 19 Paralelamente sobre todo en medio de la Pandemia del COVID 19 hemos estado viendo nuevos usos para esos mismos materiales recuperados, destinados a una población con distintas necesidades, ya no con la urgencia de vivienda y techo sinó como complementos, mobiliario y/o construcciones menores. Que no obstante pueden permitir una salida laboral viable.

Dadas las circunstancias del año 2020 y el presente año, ante la imposibilidad de actividades presenciales, se propiciaron otros usos para los materiales en estudio, desarrollos menores desde el punto de vista arquitectónico, mobiliario, complementos, construcciones accesorias, que pudieran ser materializadas en forma unipersonal.

Credito de fotografías e Imágenes, Propias

\section{BIBLIOGRAFÍA}

General y Formativa.

Acosta Wladimiro, (1976) Vivienda y clima, Ed. Nueva Visión.

Acosta Wladimiro, (1947) Vivienda y ciudad, Ed. Anconada-

Almeida Curth, Daniel, (2002) Emoción y Significado en la Arquitectura, Ed. Kliczkowski,

Cuchi Albert, y Wadel Gerardo (2007) Guía de la eficiencia energética para los administradores de fincas, Ed. Fundacion Gas Natural España

Dover Publications inc (1978). Vacation homes and log Cabins, Ed. General Publishing Company, Ltd.

Guitelman Jorge (1997), Nuevos trabajadores del turismo, CIC, Ed. Ferrograf.

Gusowsky Mari (2010) Arquitectura contemporáneas, Energía cero, Ed.Blume.

Hanono Miguel (2001), Construcción en Madera, Cima, Producciones Gráficas y Editoriales.

Jodidio Filip (2012) Arquitectura ecológica hoy, Ed.Taschen.

Krier Rob, (1983) El Espacio urbano, Ed. Gustavo Gili.

Livingston Rodolfo, (2001) Arquitectos de familia, 4ta Edición, Ed. Nobuko.

MacDonald S.O., Myrman Matts, (2007) Edifique con Fardos, ed Nobuko.

Sacriste Eduardo, (1968), Qué es la casa, Ed. Columba.

VillarroeMelvin I, (1996) Arquitectura del Vacío, Ed. Gustavo Gili. 